\title{
Pascal Quignard. La littérature à son Orient, éds. Christian Doumet et Midori Ogawa
}

\section{Stefano Genetti}

\section{(2) OpenEdition}

1 Journals

\section{Edizione digitale}

URL: http://journals.openedition.org/studifrancesi/4484

DOI: $10.4000 /$ studifrancesi. 4484

ISSN: 2421-5856

\section{Editore}

Rosenberg \& Sellier

\section{Edizione cartacea}

Data di pubblicazione: 1 settembre 2016

Paginazione: 368-369

ISSN: 0039-2944

\section{Notizia bibliografica digitale}

Stefano Genetti, « Pascal Quignard. La littérature à son Orient, éds. Christian Doumet et Midori Ogawa », Studi Francesi [Online], 179 (LX | II) | 2016, online dal 01 septembre 2016, consultato il 18 septembre 2020. URL : http://journals.openedition.org/studifrancesi/4484 ; DOI : https://doi.org/10.4000/ studifrancesi.4484

Questo documento è stato generato automaticamente il 18 settembre 2020.

\section{(c)}

Studi Francesi è distribuita con Licenza Creative Commons Attribuzione - Non commerciale - Non opere derivate 4.0 Internazionale. 


\title{
Pascal Quignard. La littérature à son Orient, éds. Christian Doumet et Midori Ogawa
}

\author{
Stefano Genetti
}

\section{NOTIZIA}

Pascal Quignard. La littérature à son Orient, sous la direction de Christian DOUMET et Midori OGAWA, Saint-Denis, Presses Universitaires de Vincennes, 2015, «L'imaginaire du texte», $190 \mathrm{pp}$.

1 Da ultimo, uno dei contes inanellati in forma di sonata in Princesse Vieille Reine (Paris, Galilée, 2015), il testo recentemente messo in scena dall'attrice Marie Vialle, testimonia dell'attrazione che le letterature e le civiltà orientali da sempre esercitano su Pascal Quignard, la cui opera, ricchissima di riferimenti a epoche e culture lontanissime fra loro eppure incessantemente intrecciate e sovrapposte, dis-orienta appunto, come osserva Christian DOUMET nello scritto introduttivo (Le plus profond, le plus lointain, pp. 9-12), ogni coordinata spazio-temporale mettendo costantemente in discussione «le plus grand lieu commun occidental, celui qu'on nomme précisément "l'Occident"» (p. 10). Alle fonti, cinesi e giapponesi soprattutto, e più in generale all'assimilazione della tradizione orientale e al ripensamento della tradizione occidentale che tale innutrizione comporta, è dedicato, almeno in parte, ciascuno dei saggi che compongono questo volume, nato dalla giornata di studio organizzata da Midori Ogawa nel novembre 2013 presso la Maison franco-japonaise di Tokyo (e in Giappone insegnano molti degli studiosi che vi hanno contribuito).

Dalla traduzione dal cinese del testo di Kong-souen Long Sur le doigt qui montre cela, prendono le mosse tanto le riflessioni di Christian DOUMET su La fascination de l'incompréhensible (pp. 39-48) - interiorizzazione dell'alterità che ne accentua l'ermetismo fino a elaborare una lingua leggendaria, come sottolinea Bénédicte 
GORRILLOT in merito all' Alexandra (Pascal Quignard et Lycophron: l'art d'un traduire paradoxal, pp. 49-66) - quanto l'ampia e coinvolgente meditazione di Mireille CALLEGRUBER sulla sagesse $d u$ vide, saggezza dell'abbandono e della benefica dispersione della coscienza soggettiva che l'opera emana (Écrire avec les mots des morts. Ne rien assagir. Les sagesses de Pascal Quignard, pp. 99-115). In conclusione del suo saggio, M. Calle-Gruber evoca lo spettacolo Medea, in collaborazione con la sciamana della danza butô Carlotta Ikeda; a questa "danza delle tenebre" Pascal Quignard associa la propria concezione dello scrivere così come la manière noire che, nell'arte dell'incisione, fa sì che l'immagine emerga dall'oscurità da cui trae origine. Analogamente, è alla pittura orientale in quanto arte del tratto che Keling WEI riconduce la figura di Meaume, l'incisore dal viso sfigurato protagonista del romanzo Terrasse à Rome, in quanto autoritratto dell'artista ( $\mathrm{La}$ (dé)figure de l'artiste, pp. 69-82).

Se Eberhard GRUBER propone una lettura decostruzionista, basata sull'ombra perturbante della verità intesa come parte costitutiva della verità, del merveilleux praticato da Quignard all'intersezione di reale e immaginario, di favoloso e mostruoso (Réécriture du conte? Ou de la latence du genre, pp. 82-98), il variegato e onnipresente bestiario quignardiano - predatori e animali domestici, cinghiali e cavalli, rane e gatte è passato in rassegna e indagato, secondo prospettive differenti, da Michaël FERRIER ( $L a$ part du chien: des esprits animaux dans l'œuvre de Pascal Quignard, pp. 117-130) e da Yann MÉVEL (L'homme et l'animal dans l'imaginaire de Pascal Quignard, pp. 131-146). Non solo l'erudizione è mobilitata da Quignard in quanto fattore di destabilizzazione dei vari saperi e dei discorsi sistematici, dogmatici e didattici che li veicolano, ma la contestazione di ogni gerarchia antropocentrica è tesa alla denuncia del retroterra bestiale della storia, al disvelamento dell'animalità originaria che portiamo, indomabile, in noi. Mentre Midori ogAWA risale alle origini della scrittura ideografica e articola tra loro la filiazione della letteratura giapponese dalla lingua cinese e la nozione di ritorno in quanto perno di una visione sciamanica e regressiva del fare letterario (L'ode de Pascal Quignard, pp. 23-37), tanto Thierry MARÉ (Pascal Quignard et la liste des commissions, pp. 147-158) quanto Vincent Giraud (La cueillette du sens. Pascal Quignard et Sei Shonagon, pp. 159-170) si soffermano sulle strategie dell'enumerazione nel romanzo Les Escaliers de Chambord, ad esempio - e sulla poetica della sensazione e della minuzia, dello scarto e della trouvaille, a partire dalle Note del guanciale della dama di corte dell'xi secolo Sei Shonagon, frammentario modello pseudo-diaristico delle Tablettes de buis d'Apronenia Avitia, un po' come l'ombra del retore latino Albuzio è evocata da Quignard, nell'estate del 1989, mentre siede su una panchina nei giardini imperiali di Tokyo («Avertissement» premesso al "romanzo di romanzi romani" Albucius).

4 «L'écrit dans le livre fait entrer son lecteur dans un passé qui n'a pas été vécu par lui. L'écrit ouvre la porte à un passé au-delà du passé» (p. 20), scrive Quignard nell'inedito Le mot littérature est sans origine (pp. 13-20) che apre il volume. Nell'interrogare la storia della parola littera, l'autore illumina il legame tra frammentazione letterale e scrittura letteraria, da un lato, e libera associazione in psicanalisi, dall'altro. In conclusione - e quasi a illustrare la saggezza del vuoto e dell'abbandono cui si è fatto cenno - è riportata invece la condensazione del romanzo Villa Amalia in otto brevi sezioni scandite da riduzioni di opere di Schubert, Haydn, Beethoven, Couperin, Purcell, Gluck e Bach e da composizioni di Toru Takemitsu e Toshio Hosakawa, eseguite dalla pianista Kaorou Hakata in occasione del récit-récital intitolato Femme disant adieu: «Tous les 
deux», afferma lo scrittore, «Madame Kaorou Hakata et moi, on a crypté nos vies à l'intérieur de ces fragments». 